\title{
A 5-GHz Combined Oscillator/Mixer
}

\author{
Chris van den Bos ${ }^{(1)}$, Luís Bica Oliveira ${ }^{(2)}$, Jorge R. Fernandes ${ }^{(2)}$, and Chris J. M. Verhoeven ${ }^{(3)}$ \\ (1) Systematic Design, Delft, The Netherlands \\ (2) I.S.Técnico/INESC-ID, Lisboa, Portugal. \\ (3) TU Delft, Electronics Research Laboratory/DIMES, The Netherlands. \\ c.vdbos@systematic.nl, \{luis.b.oliveira;jorge.fernandes\}@inesc-id.pt, c.j.m.verhoeven@its.tudelft.nl
}

\begin{abstract}
In this paper an IQ oscillator/mixer circuit is presented in which the mixers are an integral part of the oscillator. The oscillator alone has accurate quadrature outputs, even in the presence of component mismatch. This precise quadrature is preserved when mixers are incorporated in the oscillator. An oscillator/mixer core for a carrier frequency of $5 \mathrm{GHz}$, suitable for a low-IF front-end, was designed and tested. The power consumption is $30 \mathrm{~mW}$ from a $2.5 \mathrm{~V}$ supply, and a phase error of $1^{\mathrm{o}}$ was measured.
\end{abstract}

\section{INTRODUCTION}

In modern radio receiver front-ends generation of accurate quadrature signals for down-conversion is required. In direct-conversion front-ends, errors in the quadrature relationship lead to cross-talk between the in-phase and the quadrature components of the received signal. In combination with additive noise, this cross-talk increases the bit error rate. In front-ends with a non-zero intermediate frequency (IF), inaccurate quadrature (due to mismatch) leads to a finite image rejection. The severity of this is dependent on the properties of the signals at the image frequency, which are difficult to assess at design time. Therefore a safety margin must be used.

Several solutions for these problems have been proposed. They are based on either filtering or on error compensation. Filtering relies on suppressing the signals at the image frequency before frequency conversion takes place. In case of a receiver with a low IF [1], this approach is not possible due to the extreme selectivity required of the image-reject filter. Error compensation consists of estimating the error during operation and compensating it by adjusting the carrier phases and amplitudes. Systems for doing this are rather complicated and their performance depends on the actual received signals (e.g. [2]).

In this paper, a solution is proposed which is based on a cross-coupled relaxation oscillator [3], which generates signals that are inherently in quadrature [4]. If mixers were added outside this oscillator, the mismatch of the circuit components in the mixer would cause a loss of accuracy, and not much could be won. Therefore, it is proposed that the mixers be made an integral part of the oscillator.

This work was supported by POSI and the Portuguese Foundation for Science and Technology (FCT) through project POCTI /38533/ESE/2001 and scholarship BD 10539/2002, and through the UbiCom program (Delft University of Technology, 2002). The integrated circuit was designed and processed as part of the SRC SiGe Design Challenge.
The structure of this paper is as follows. In section II the combined oscillator/mixer is proposed. In section III the Low-IF front-end is described and in section IV the circuit schematics are presented. In section $\mathrm{V}$ the measurement results are presented, and the conclusions are drawn in section VI.

\section{Proposed Oscillator/Mixer ArChitecture}

A block diagram of a cross-coupled relaxation oscillator is depicted in Fig. 1. It consists of two relaxation oscillators that determine the switching instants of each other's Schmitt-triggers, thus causing the output signals to be in quadrature. A detailed description of the oscillator behaviour is available in $[3,5]$.

Mismatch between circuit components causes a deviation from the ideal $90^{\circ}$ phase difference between the output signals of the Schmitt-triggers. In $[3,5]$ it is shown that this deviation is a second-order, rather than first-order effect. Therefore, the circuit is inherently less sensitive to errors than, e.g., ring oscillators, polyphase filters, or flip-flop based quadrature oscillators. This low sensitivity might be exploited in the design of quadrature modulators/demodulators. However, offset and mismatch in the mixers outside the oscillator degrade the quadrature relationship, and the potential advantage over the other quadrature-generation schemes is reduced.

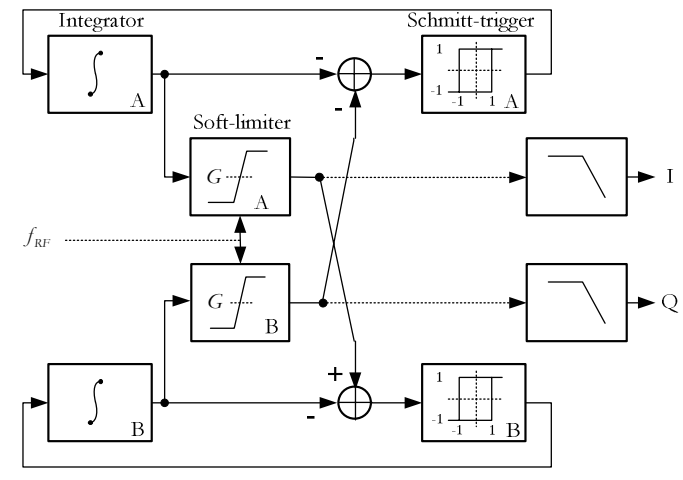

Figure 1. Block diagram of the cross- coupled oscillator/mixer in demodulation configuration. 
This problem can be overcome by including the mixing function in the oscillator, thus preserving the low sensitivity. The inclusion of mixing should not affect the basic operation of the oscillator.

The oscillator consists of several functional blocks with parameters as indicated in Fig. 1. Inclusion of the mixing function means making one of these parameters the product of a constant and the signal to be mixed. A parameter must be chosen that when varied (within certain boundaries of course) does not affect the oscillator operation. It is to be expected that introducing mixing in a relaxation oscillator always affects a parameter that influences the oscillation frequency. The only suitable location for injection of the mixing signal is the cross-coupling paths. It can be shown that the oscillator performance is not affected by injecting the RF signal using variable $G$ in Fig. 1, which causes the gain of the soft limiter to change, but has no influence on the zero-crossing moments of the soft-limiter output signal. Either the gain or the clipping levels of the soft-limiter may be affected, but, since this does not change the zerocrossings of the signal at the output of the soft-limiter (which are responsible for the quadrature relationship and frequency of the oscillator [6]), the oscillator performance is not impaired, as long as the soft-limiter gain does not become zero or change sign.

\section{LOW-IF FRONT-END}

The block diagram of the low IF front-end in which use is made of accurate quadrature mixing at a high frequency is shown in Fig. 2. In a full receiver, the two downconverted signals can be digitized and mixed digitally for the second time.

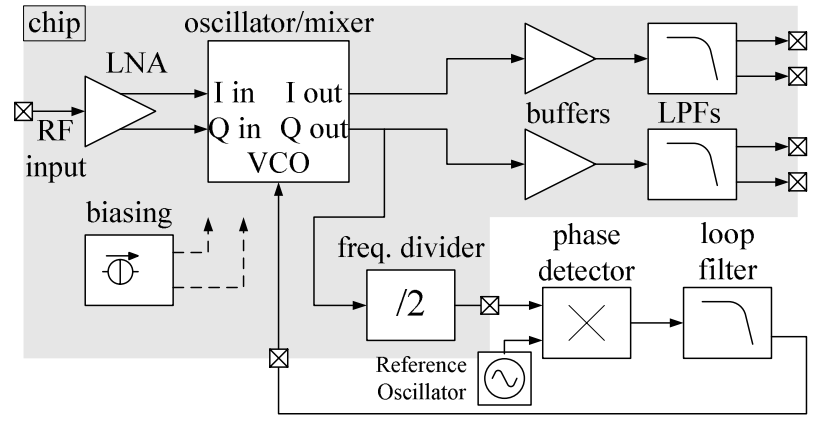

Figure 2. Oscillator/Mixer in its test environment

The proposed IQ oscillator-mixer converts an RF signal to a low IF (in this case $10 \mathrm{MHz}$, just high enough to contain one channel of an OFDM signal in the IEEE 802.11a standard). After the oscillator/mixer, the I and $\mathrm{Q}$ output signals are filtered to remove high-frequency components, and buffered, so that off-chip loads can be driven. The two IQ outputs are connected directly to the measurement equipment and a measurement of the quadrature relationship is done at a low frequency, which is easier.
To drive the oscillator/mixer, a low-noise amplifier (LNA) with $50 \Omega$ input impedance is used. To reduce the close-in phase noise, the oscillator/mixer is included in a PLL. To keep the chip complexity as low as possible, the phase detector and loop filter are connected externally and to minimize cross-talk from the oscillator signal to the LNA input, the oscillator output signal is divided by 2 in frequency before it is brought off-chip. Since the oscillator/mixer is easily tuned, it is also sensitive to noise. Therefore, an on-chip bias block is used.

\section{Circuit Schematics}

The circuit was designed in IBM's SiGe Blue Logic BiCMOS 6HP technology. This technology includes 47-GHz NPN transistors and $0.25 \mu \mathrm{m}$ MOS transistors. The circuits have a $2.5 \mathrm{~V}$ supply voltage.

\section{A. LNA}

The purpose of the LNA is to convert an input signal into tail currents for the limiters in the oscillator/mixer circuit. The LNA is kept as simple as possible, since the main objective of the design is to demonstrate the possibilities of the oscillator/mixer. The schematic of the LNA is shown in Fig. 3. It consists of two inductively degenerated common emitter stages in parallel.

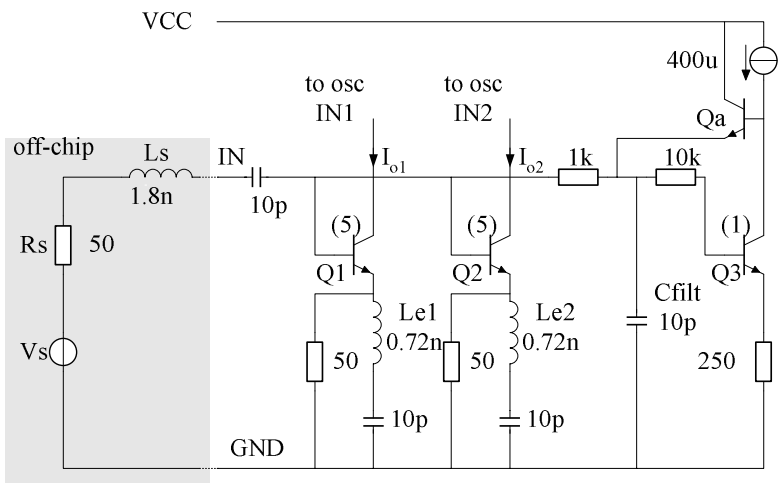

Figure 3. Schematic of the LNA.

Accurate collector current matching is achieved by resistive emitter degeneration at DC. The degeneration is bypassed at the frequencies of interest using a $10 \mathrm{pF}$ capacitor in series with $L_{\mathrm{e} 1}$ and $L_{\mathrm{e} 2}$. The current itself is derived by mirroring a smaller bias current. ESD input protection diodes and pad models have been incorporated for the simulation. The noise figure is about $2.5 \mathrm{~dB}$ and the transcondutance is about $25 \mathrm{~mA} / \mathrm{V}$. The simulated IIP3 is $+7 \mathrm{dBm}$.

\section{B. Oscillator/Mixer}

The oscillator/mixer has been implemented using a common cross-coupled relaxation oscillator topology [4], as shown in Fig. 4. The tail currents of the limiters in the cross-coupling paths are supplied by the LNA. In this way, the gain of these limiters is modulated by the LNA input 
signal, and mixing is performed. The implemented circuit has 27 elements and an overall area of $250 \times 100 \mu \mathrm{m}^{2}$. The implementation uses $\mathrm{R}=50 \Omega, \mathrm{C}=300 \mathrm{fF}$, and the bias currents are $2 \mathrm{~mA}$ for the oscillator core transistors and $2 \mathrm{~mA}$ for the tail currents of the soft-limiters. Terminal IBIAS in Fig. 4 is connected to a pad, in order to tune the oscillator. The tuning range is about $1 \mathrm{GHz}\left(20 \% f_{\text {osc }}\right)$. All the transistors were dimensioned and biased to have the highest $f_{\mathrm{T}}$ possible in the process.

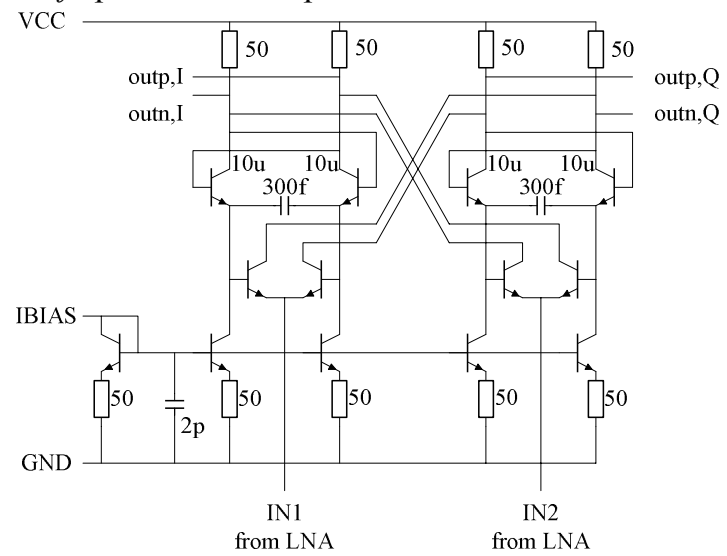

Figure 4. Schematic of the IQ oscillator/mixer.

\section{Other circuits}

The frequency divider divides the oscillator signal frequency by 2 , so that it is in a frequency band different from that of the front-end input signal and can be brought off-chip for use in a frequency synthesizer. It is implemented as a current-mode logic D-type flip-flop, the outputs of which are connected to the inputs. Current-mode logic has been chosen since this logic family creates ideally no spikes on the supply voltage, due to the constant current biasing. The total bias current is $3.4 \mathrm{~mA}$ and simulations showed reliable division for input signals up to $10 \mathrm{GHz}$.

Phase noise simulations have been performed and with the aforementioned input signal, the phase noise floor was at about $-144 \mathrm{dBc}$, which is below the value expected from the oscillator output signal.

An on-chip bias current generator is used in this design to avoid the design of separate bias current generators for each circuit. A PTAT current source is used, generating a current equal to the ratio of the thermal voltage and a resistor value. To reduce the temperature dependence to some extent, a resistor with a positive temperature coefficient was used. There are no connections off-chip to sensitive parts of the bias current generator, which further reduces sensitivity to noise.

The divider buffer converts the differential output signal of the divider into a single (open-collector) output for a $50 \Omega$ load, over which a voltage of $300 \mathrm{mV}_{p p}$ is generated (about $-4 \mathrm{dBm}$ first harmonic power).

The signal buffer is a common-collector stage followed by a low pass filter. The low pass filter is a $5^{\text {th }}$ order LC Butterworth filter with a cutoff frequency of $1 \mathrm{GHz}$. For matching purposes, the filters are also on-chip. In a normal implementation, these filters would be replaced by much smaller active filters. In this application, it is more important to avoid noise and distortion.

A photo of the chip is shown in Fig. 5.

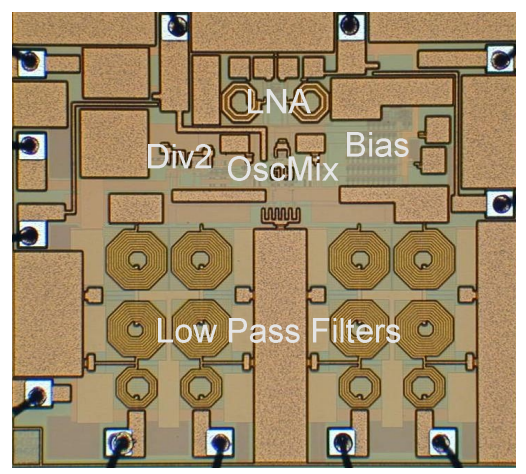

Figure 5. Die Photo (2.0 mm x 2.4mm).

The remaining external blocks to build the Phaselocked-loop (PLL) are the phase detector and the loop filter. These blocks are connected externally. This was decided because of time limitations and to concentrate efforts on the design of the oscillator-mixer. For the phase detector, a commercial mixer (Miteq DMX0418L) is used, which can be used up to $18 \mathrm{GHz}$. The mixer is followed by a first-order filter with an input impedance of $50 \Omega$, that has one pole and one zero. Since the oscillator frequency is controlled by a current, the filter is followed by a simple transconductance amplifier with a gain of about $1 \mathrm{~mA} / \mathrm{V}$. A signal-independent current, for tuning the oscillator to its nominal value, can be set by means of a potentiometer.

\section{MEASUREMENTS}

\section{A. Measurement setup}

The measurement setup is shown in Fig. 6. The transformers convert the balanced output signals from the chip to single-ended signals, suitable for measurement by an oscilloscope. To avoid interference through the power supply, a battery supply was used. All parts were mounted inside an aluminium box, and signals were led in and out of the box by means of BNC or SMA connectors only.

The quadrature relationship between the outputs is measured by applying a single-carrier signal to the LNA and comparing the phases of the two output signals. The oscillator/mixer is phase-locked to a reference signal from a generator, to prevent the frequency of the oscillator/mixer from drifting. The output signals are fed to a digital oscilloscope, where they are stored for computer analysis. 


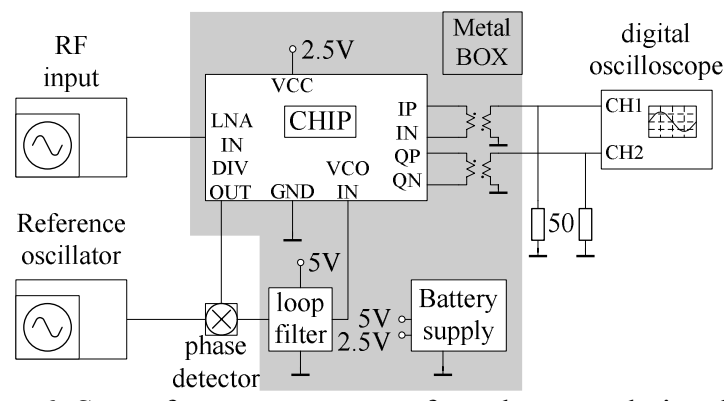

Figure 6. Setup for measurement of quadrature relationship.

Since the phase errors to be measured are very small, it is necessary to avoid extra phase errors due to path length differences from oscilloscope outputs to the inputs. At a frequency of $10 \mathrm{MHz}$, the output frequency at which the measurements were performed, the free-space wavelength is $30 \mathrm{~m}$; taking into account the effect of dielectric of the coaxial cables, the wavelength is about $15 \mathrm{~m}$. A path length difference of $4.2 \mathrm{~mm}$ then yields a phase difference of $0.1^{\circ}$. For this reason, care has been taken to match the path lengths accurately. The paths on the PCB are matched by careful layout. The link between the PCB and the transformers is through connectors, which can be swapped so that path length differences can be distinguished from actual quadrature errors.

The oscillator phase noise has been measured using an HP3048A phase noise measurement system, while the PLL is in lock and in the absence of an input signal to the LNA. The PLL is kept locked, since the drift of the carrier frequency of the oscillator/mixer when free-running proved too much for the phase-noise measurement system. As the bandwidth of the PLL is small, the phase noise measurement is determined by the oscillator from about $1 \mathrm{MHz}$ of offset frequency onward.

\section{B. Measurement results}

Since a precise quadrature relationship is expected, visual inspection of the waveforms does not suffice. The phase relationship between two sinusoidal signals $x$ and $y$ with identical periods can be determined by using their (vector) inner product. This method of determining the phase between signals is robust to additive noise, including quantization noise of the digital oscilloscope.

The stored waveforms have first been truncated to contain an integer number of periods. Then, any offset was removed and the amplitude was normalized to 1 . Finally, the quadrature error was determined.

The measured quadrature errors are about $1^{\circ}$ in 4 tested samples. This phase error is larger than expected. Furthermore, the amplitude of the output signals was smaller than expected. This may be explained by the fact that in the simulations, flip-chip gold pads were used, which were later found not to be available in the particular chip fabrication run used. This affects the performance of the LNA. In a future chip, the LNA should be redesigned to improve the input matching and gain. This will increase the output signal amplitude and increase the accuracy in the measurement of the quadrature errors.

The measured oscillator phase noise is shown in Fig. 7. Since this noise has been measured after frequency division by two, $6 \mathrm{~dB}$ must be added to find the actual phase noise of the oscillator/mixer (assuming a noiseless frequency divider). Phase noise close to the carrier is suppressed by the PLL. The PLL bandwidth is about $100 \mathrm{kHz}$. Above $1 \mathrm{MHz}$, a slope of $-20 \mathrm{~dB} /$ decade is observed. The phase noise of the oscillator at $10 \mathrm{MHz}$ offset from the carrier is $-114 \mathrm{dBc} / \mathrm{Hz}$, which is a typical value for this type of oscillator.

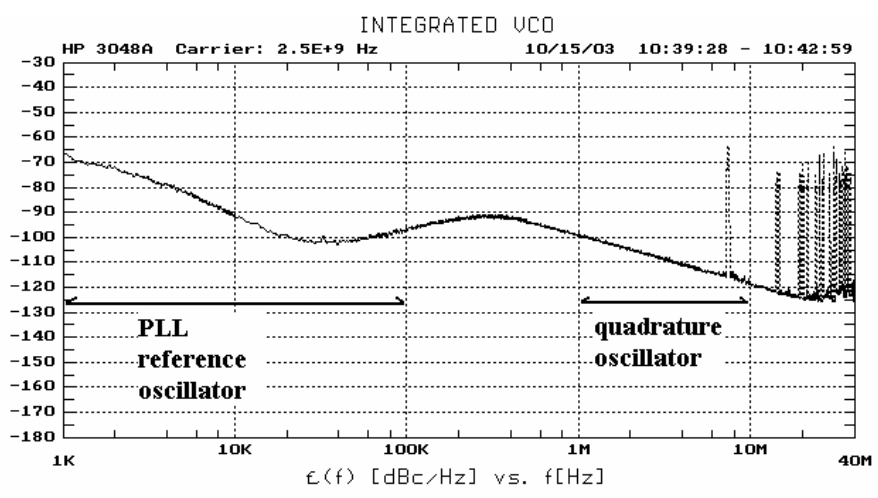

Figure 7. Measured phase noise of the oscillator/mixer.

\section{CONCLUSIONS}

A new architecture to obtain quadrature mixing within the cross-couple path of an IQ oscillator has been presented. An integrated oscillator/mixer in a low IF architecture has been designed and tested. Measurements confirm that this class of oscillators can perform wideband mixing without the need for separate mixers that would degrade the quadrature relationship. A phase error of about $1^{\circ}$ for the oscillator/mixer block has been found.

\section{REFERENCES}

[1] J.Crols and M. Steyaert, CMOS Wireless Transceiver Design, Kluwer Academic Publishers, 1997

[2] L. Der and B. Razavi, "A 2GHz CMOS Image-Reject Receiver with LMS Calibration", IEEE J. Solid-State Circ., vol.38, pp. 167-175, Feb. 2003.

[3] C.J.M. Verhoeven "A High-Frequency Electronically Tunable Quadrature Oscillator", IEEE J. Solid-State Circ., vol.27, pp. 10971100, July 1992.

[4] J.Fernandes, M.Kouwenhoven, C. van den Bos, K. van Hartingsveldt, and C.Verhoeven, "A 5.8-GHz Quadrature Cross-Coupled Oscillator", ISCAS’02, vol. II, pp.404-407 May 2002.

[5] J.Fernandes, M.Kouwenhoven, and C. van den Bos, "The Effect of Mismatch and Disturbances on the Quadrature Relation of a CrossCoupled Relaxation Oscillator", ISCAS'01, vol. I, pp.476-479, May 2001.

[6] M. Kouwenhoven, C. van den Bos, M. van Nieuwkerk and C. Verhoeven, "Quadrature Modulator and Demodulator", Patent $\mathrm{Nr}$. PCT/NL02/00060, Jan. 2002. 